\title{
Autoconceito dos professores: principais factores usando modelos de Análise de Dados Multivariada
}

\section{Teachers' self-concept : finding main factors and clusters by EDA models}

\author{
Vitor Franco* \\ Helena Bacelar-Nicolau**
}

\begin{abstract}
RESUMO
$\mathrm{O}$ autoconceito tem sido considerado uma dimensão muito importante da personalidade do professor, da sua prática e do seu desenvolvimento pessoal (MARKUS; WURF, 1987; SIMÕES, 2001).A investigação que apresentamos foi efectuada com 281 professores de Ciências da Natureza, do terceiro ciclo do Ensino Básico, em Portugal, usando o ICAC- Inventário Clínico do Auto Conceito (VAZ-SERRA, 1986). Na análise dos dados obtidos foram usados diferentes métodos de Análise Multivariada, apresentando-se os resultados da análise factorial de correspondências e nos modelos de classificação hierárquica baseados no coeficiente de afinidade. Os resultados obtidos: 1) confirmam a importância de dois grandes factores presentes no Autoconceito: aceitação social e auto-eficácia; 2) caracterizam estes principais factores no que se refere ao Autoconceito clínico dos professores; 3 ) mostram como esses factores são determinantes na forma como cada professor constroi o seu autoconceito.
\end{abstract}

Palavras-chave: autoconceito; professores; auto-eficácia; aceitação social.

* Universidade de Évora. Professor Associado do Departamento de Psicologia

** Universidade de Lisboa. Professora Catedrática da Faculdade de Psicologia e Ciências da Educação. 


\begin{abstract}
The self-concept has been considered as a very important dimension on teacher's personality, practice and development (MARKUS; WURF, 1987; SIMÕES, 2001). The present research concerns a sample of 281 teachers of Natural Science of the Third Cycle of Basic Education from Portugal that responded to the I.C.A.C. - Self-Concept Clinical Inventory (VAZSERRA, 1986). In the analysis of the questionnaires different multivariate data analysis methods have been used. This paper describes some results issued from correspondence analysis and hierarchical clustering models based on the affinity coefficient. The results obtained: 1) confirm the importance of two general main factors / types which are present in selfconcept: social acceptance and self-efficiency; 2) characterise these main factors when teachers' clinical self-concept is concerned and 3) show how determinant these factors are for the building of self-concept that allow us to differentiate teachers.
\end{abstract}

Keywords: self-concept; teachers; self-efficiency; social acceptance.

\title{
Introdução
}

O autoconceito é um constructo teórico util e fecundo na pesquisa psicológica, apesar da atenção que lhe tem vindo a ser dada no campo do desenvolvimento e prática dos professores ser surpreendentemente escassa (ROCHE; MARSH, 2000).

$\mathrm{O}$ avanço da investigação nesse domínio tem mostrado que, embora não possa mais ser entendido como uma entidade estática e invariável (MARKUS; WURF, 1987), o autoconceito se revela útil como elemento antecipador, dandonos "a noção da continuidade e da identidade do ser humano no tempo, ajudandonos a conhecer e a predizer os seus comportamentos, independentemente de eventuais influências do meio ambiente" (VAZ-SERRA, 1988, p.128).

$\mathrm{O}$ autoconceito refere-se à forma como o indivíduo vê a si próprio e, embora de algum modo dependente das condições em que se encontra, depende dos motivos pessoais presentes, o que lhe dá um carácter de estabilidade e unidade no tempo e no espaço. Está implicado em todos os processos de organização e processamento da informação relevante para o indivíduo e na forma como organiza os seus comportamentos.

A génese do autoconceito encontra-se ligada a quatro grandes ordens de 
factores (VAZ-SERRA,1988): a apreciação dos outros sobre o comportamento do sujeito; a forma como ele age em situações específicas e a recordação que tem desse desempenho; a comparação do seu próprio comportamento com o do grupo social de pertença e o confronto do comportamento individual com os valores instituídos por um grupo normativo, ou seja, a forma como o indivíduo considera a sua conduta face a uma moral.

O autoconceito relaciona-se com a identidade, com o conteúdo das percepções e crenças do indivíduo acerca de si próprio, e com a auto-estima. Está ligado a traços de personalidade, pelo que permite compreender a continuidade $\mathrm{e}$ a coerência individuais e ajudar a predizer as acções. Permite pois compreender porque é que o indivíduo inibe ou facilita um dado comportamento.

No que se refere à importância do autoconceito relativamente aos professores, tem sido estudada especialmente a sua ligação com o desenvolvimento e sucesso educativo dos alunos (CHAN et al, 1992), com ambiente educativo e da sala de aula (TONELSON, 1981; SCHULTZ; WOLF, 2006; SIMÕES, 2001) e ainda o seu papel no bem-estar emocional dos professores (VILLA; CALVETE, 2001).

A avaliação do autoconceito, feita através do ICAC - Inventário Clínico do Autoconceito, dá-nos uma dimensão clínica do autoconceito que se distingue do autoconceito académico abordado em múltiplos estudos. Não se centra especificamente nas dimensões relativas à profissão ou à prática profissional mas, posicionando-se numa perspectiva clínica, procura ter em consideração o individuo de um modo uno e completo.

Desde a construção do ICAC, a avaliação clínica do autoconceito tem sido utilizada num vasto conjunto de investigações acerca de diferentes dimensões psicológicas. $\mathrm{O}$ autoconceito, assim avaliado, parece ter uma correlação negativa elevada com a ansiedade social (VAZ-SERRA; GONÇALVES; FIRMINO, 1986). Estudos relacionando o autoconceito com o stress (VAZ-SERRA; RAMALHEIRA; FIRMINO, 1988; VAZ-SERRA, 1992), mostram que os indivíduos com autoconceito elevado e sentimentos de auto-eficácia tendem a apresentar bons mecanismos para lidar com situações indutoras de stress. $\mathrm{O}$ autoconceito e os mecanismos de coping parecem ser bons preditores do estado de saúde mental.Vaz-Serra, Matos e Gonçalves (1986) verificaram também existir uma correlação significativa entre os valores de autoconceito e os resultados obtidos no Inventário Depressivo de Beck, concluindo que quanto melhor o autoconceito menor a tendência para o aparecimento de sintomas depressivos, sendo que os valores de autoconceito assim obtidos mostram ter boa capacidade preditiva em relação à variável depressão.

As pessoas com autoconceito mais elevado têm mostrado maior tendência para desenvolver expectativas positivas (e vice-versa), mostrando o ICAC uma 
boa capacidade preditiva em relação às expectativas (VAZ-SERRA; ANTUNES; FIRMINO, 1986).

No que se refere às atribuições, um outro estudo feito com o mesmo instrumento (VAZ-SERRA, 1988) mostrou que os indivíduos com autoconceito elevado tendem a atribuir o êxito a factores internos e os fracassos a factores externos.

Faria (1993) evidencia uma relação entre autoconceito, comunicação individual e social, temperamento, apoio social e êxito. $\mathrm{O}$ autoconceito relaciona-se de modo bastante significativo com sociabilidade, emocionalidade e medo, no primeiro caso de modo positivo e nos restantes de modo negativo. Ao considerar os factores do autoconceito, a autora verificou que a auto-eficácia se correlaciona negativamente com o temperamento global, enquanto que tanto a auto-eficácia quanto a aceitação social (bem como o autoconceito global) se correlacionam negativamente com a emocionalidade e com o medo. Tudo isso aponta para que o indivíduo com bom autoconceito possa ter um melhor relacionamento interpessoal. A importância do autoconceito é, pois, central na compreensão da comunicação intraindividual e intersocial.

Nos professores o autoconceito é uma dimensão fundamental para compreendermos a forma como agem profissionalmente e como organizam a sua relação e comunicação com os alunos. Daqui a importância do presente estudo, que visa compreender como se organiza o autoconceito numa população específica de professores, quais os factores que o compõem, obtendo assim informação que nos permita ter em conta a variabilidade inter-individual nessa dimensão.

\section{Metodologia}

\section{População e amostra}

O presente estudo foi efectuado com uma amostra nacional, aleatoriamente constituida de 281 professores de Ciências da Natureza, proveniente de 65 . 20,8 \% são do sexo masculino e 79,2 do sexo feminino, reflectindo a conhecida tendência para a feminização das actividades de ensino.

No que se refere às idades, $12,7 \%$ dos professores têm menos de 25 anos; $40,1 \%$ entre 25 e 34 anos; $27,8 \%$ entre 35 e 44 anos; $14,4 \%$ entre 45 e 54 anos e apenas $4,9 \%$ têm idade igual ou superior a 55 . Estamos pois perante uma população maioritariamente jovem, em que mais da metade tem idade inferior a 35 anos. 
No que se refere à categoria profissional, o número de professores profissionalizados representou $84,2 \%$ do total.

No que se refere ao tempo total de serviço docente verificou-se que $32,4 \%$ têm menos de 5 anos como professor, o que nos dá a ideia de estarmos perante uma população jovem com muitos indivíduos em fase inicial de carreira: 17,6 $\%$ têm entre 5 e 9 anos; $19,4 \%$ têm de 10 a 14 anos; $13 \%$ têm de 15 a 19; 8,5 $\%$ têm entre 20 e 24 e $9,2 \%$ têm 25 ou mais anos.

\section{Instrumento utilizado: Inventário Clínico do Autoconceito}

O Inventário Clínico do Autoconceito foi criado por Vaz-Serra em 1985, referindo-se apenas aos aspectos sociais e emocionais considerados importantes no ajustamento pessoal.

Trata-se de uma escala, de tipo Likert, de auto-avaliação, em que é apresentado aos sujeitos um questionário, com 20 questões que devem ser respondidas segundo cinco modalidades: não concordo, concordo pouco, concordo moderadamente, concordo muito e concordo muitíssimo. Cada modalidade é codificada de 1 a 5, com excepção de três itens em que, por estarem redigidos de forma negativa, as pontuações devem ser revertidas.

Ao registar as percepções que cada indivíduo tem de si próprio, este instrumento considera o autoconceito como uma medida de traço de personalidade, de modo que os resultados obtidos permitem afirmar algo sobre o indivíduo. $\mathrm{O}$ ICAC assume que a forma como o indivíduo avalia a si próprio é importante na maneira como vê o mundo e se relaciona com os outros.

A resposta a todas as questões permite a obtenção de uma nota global, bem como vários índices sobre a pessoa. Pela maneira como está construído, o valor global do autoconceito é dado pela soma das pontuações obtidas, de modo que uma pontuação mais elevada corresponde sempre a um melhor autoconceito.

Todos os itens têm bom valor discriminativo, não sendo sensíveis à variável sexo. O Inventário tem boa consistência interna (0.791 Coeficiente SpearmanBrown) e elevada estabilidade temporal (com coeficiente de correlação de teste-reteste de 0.838). A validade preditiva é boa, tendo sido corroborada por investigações posteriores (VAZ-SERRA; GONÇALVES; FIRMINO, 1986; VAZ-SERRA; MATOS; GONÇALVES, 1986).

No estudo factorial do ICAC foi encontrado um número restrito de factores que transmitem a informação presente no conjunto total das variáveis e que, no seu conjunto, explicam 53,4 \% da percentagem cumulativa da variância.

Dos seis factores encontrados, os dois primeiros têm sido considerados como os mais relevantes: 
O primeiro, explicando $20,12 \%$ da variância, foi designado como factor de Aceitação/rejeição social. Refere essencialmente o significado que o sujeito pode ter para os outros. Para ele contribuem especialmente os itens 1- Simpático; 4- Falador; 9- Aceitável; 10- Pragmático; 17-Agradável e 16- Bem-estar ${ }^{1}$.

O segundo factor, explicando $9,01 \%$ da variância, foi designado de Auto-eficácia. Engloba a competência sentida em relação a diferentes situações: resolver problemas e dificuldades, não desistir nas tarefas, rapidez de execução, enfrentar os problemas, persistência na resolução das dificuldades, não dependência dos outros e existência de energia para ultrapassar as dificuldades. Contribuem de modo mais significativo para este factor os itens: 3- Não desistente; 5- Rápido; 8- Enfrentador; 11- Persistente; 18- Não-dependente e 20- Enérgico.

Dois outros factores foram ainda considerados: Maturidade psicológica, que é apresentado como indicando um grande sentido de responsabilidade, tolerância, franqueza na expressão dos sentimentos e gosto pela verdade, e Impulsividade/actividade. Os restantes dois factores teriam menos importância e um carácter misto.

\section{Análise de Dados Multivariada}

Para o presente trabalho procurou-se uma metodologia que permitisse compreender a estrutura subjacente ao conjunto de variáveis que queríamos considerar. Foi utilizada uma metodologia de Análise de Dados Multivariada ${ }^{2}$ na qual é possível distinguir três fases (BACELAR-NICOLAU; NICOLAU, 1993): a) recolha, codificação e análise de dados; b) definição e aplicação ou ajustamento de um modelo; c) interpretação dos resultados.

A Análise de Dados Multivariada desenvolveu-se na década de 1960, baseada, segundo Giffi (1990, apud BACELAR-NICOLAU; NICOLAU, 1993, p. 522), "no estudo de sistemas de variáveis aleatórias correlacionadas ou de amostras aleatórias desses sistemas".

A Análise de Dados Multivariada (ADM) cobre um campo mais vasto e variado do que a Análise Estatística Multivariada clássica (confirmatória), diferindo na ênfase que é dada a cada uma das diferentes fases. Na análise estatística clássica o papel central está no modelo e nas inferências estatísticas, enquanto

1 Optou-se por utilizar essa forma abreviada de descrever os diferentes itens (BACELARNICOLAU, 1989) por facilitar a leitura, uma vez que a identificação numérica ou a descrição de toda a frase que constitui cada um deles seria insuficiente ou excessiva para os referirmos.

2 Para a Análise de Dados Multivariada foi utilizado o software SPAD-N e LEASP, com a colaboração do LEAD - Laboratório de Estatística e Análise de Dados da Faculdade de Psicologia e Ciências da Educação de Universidade de Lisboa. 
que na ADM são os dados que assumem o papel primordial.

É possível diferenciar duas grandes famílias de métodos de ADM que permitem fazer a redução da informação (LEBART; SALEM, 1988) e que podem ser complementares. O primeiro grupo é o dos métodos factoriais, que produz representações gráficas em que as proximidades geométricas entre pontos-linhas e entre pontos-colunas da tabela de dados traduzem as associações estatísticas entre linhas e colunas. Através da análise factorial é possível reduzir a informação contida num quadro com grande quantidade de variáveis a um número mais limitado de variáveis correspondendo aos eixos factoriais. É a essa família que pertence a Análise de Correspondências que utilizámos neste estudo.

O segundo grupo inclui os métodos de classificação que fazem o reagrupamento das linhas e/ou das colunas em classes ou famílias de classes hierarquizadas. A representação gráfica desses agrupamentos permitem-nos entender a forma como as variáveis ou os indivíduos tendem a agrupar-se tendo em conta determinado binómio coeficiente de proximidade - critério de agregação.

$\mathrm{Na}$ Análise Classificatória pretende-se agrupar um conjunto de dados em classes de elementos semelhantes ou obter uma família de classes encaixadas, representando uma certa hierarquia. A utilização de uma metodologia de Análise Classificatória Hierárquica implica, em primeiro lugar, a escolha de um índice de comparação ou coeficiente de semelhança. A construção da hierarquia de partições "assenta na escolha de um índice ou coeficiente de comparação entre pares de elementos [...] e a sua extensão a um coeficiente entre pares de classes do conjunto" (BACELAR-NICOLAU, 1980, p. 9). A semelhança entre dois indivíduos será tanto maior quanto mais próximo for o seu comportamento em relação às variáveis. Duas variáveis serão tanto mais semelhantes quanto o comportamento dos indivíduos em relação a elas for semelhante. Neste estudo foram usados o coeficiente de afinidade e o coeficiente probabilístico associado de validade da afinidade, VAL (BACELAR-NICOLAU, 1980, 1985; BACELAR-NICOLAU; DÓRIA, 1989).

A utilização de uma metodologia de Análise Classficatória Hierárquica implica, em segundo lugar, a definição de critérios de agregação adequados entre pares de classes. Foram usados neste estudo diferentes critérios de agregação: métodos de agregação clássicos, como o single linkage, SL e o complete linkage, CL, e métodos probabilísticos, como o Algoritmo de Validade da Ligação, AVL, e o Algoritmo de Validade da Ligação-Bacelar, AVB (BACELAR-NICOLAU, 1985, 1987; BACELAR-NICOLAU; NICOLAU, 1994a; LERMAN, 1981; NICOLAU; BACELAR-NICOLAU, 1982, 1998).

Uma hierarquia de classificações consiste numa sucessão de partições ou classificações encaixadas, de modo que a primeira é a partição mais fina e a última a partição em que todos os elementos estão reunidos numa única classe. 
Cada classe constitui um grupo de elementos mais semelhantes entre si do que em relação aos elementos dos outros grupos.

\section{Resultados}

\section{Valores globais do autoconceito}

O valor médio do autoconceito obtido no ICAC foi 76,9 com uma muito ligeira e não significativa variação entre homens e mulheres.

Comparando os valores obtidos por grupo etário (Quadro 1) verifica-se que o grupo em que os valores são mais elevados é o dos 25 a 34 anos, havendo a partir daí um decréscimo progressivo nas duas classes que se lhe seguem, para voltar a subir, ligeiramente, na classe dos professores com mais idade. As diferenças não são, no entanto, significativas.

Relativamente à variável tempo de serviço docente (Quadro 2) verifica-se uma tendência decrescente, observando-se os valores mais elevados no grupo de professores com menos de 5 anos $(78,01 \%)$ para ir diminuido até atingir o valor mais baixo no grupo de professores com 25 ou mais anos de serviço.

\begin{tabular}{|c|c|c|c|}
\hline & Média & Desvio Padrão & N \\
\hline Menos de 25 anos & 76,5833 & 6,2991 & 36 \\
\hline De 25 a 34 anos & 77,8596 & 5,8595 & 114 \\
\hline De 35 a 44 anos & 76,9872 & 5,5957 & 78 \\
\hline De 45 a 54 anos & 74,6500 & 5,8246 & 40 \\
\hline 55 anos ou mais & 75,9231 & 7,6427 & 13 \\
\hline Total & $\mathbf{7 6 , 9 0 7 5}$ & $\mathbf{5 , 9 8 4 4}$ & $\mathbf{2 8 1}$ \\
\hline
\end{tabular}

QUADRO 1 - MÉDIAS POR IDADES

\begin{tabular}{|c|c|c|c|}
\hline & Média & Desvio Padrão & N \\
\hline Menos de 5 anos & 78,0109 & 6,3358 & 92 \\
\hline De 5 a 9 anos & 77,0200 & 5,1961 & 50 \\
\hline De 10 a 14 anos & 75,8364 & 5,5803 & 55 \\
\hline De 15 a 19 anos & 76,8611 & 5,5916 & 36 \\
\hline De 20 a 24 anos & 76,1304 & 7,1306 & 23 \\
\hline 25 anos ou mais & 75,7600 & 6,2402 & 25 \\
\hline Total & $\mathbf{7 6 , 9 0 7 5}$ & $\mathbf{5 , 9 8 4 4}$ & $\mathbf{2 8 1}$ \\
\hline
\end{tabular}

QUADRO 2 - MÉDIAS POR TEMPO DE SERVIÇO 
Comparando os valores obtidos pelos professores profissionalizados e não profissionalizados não se encontram diferenças significativas, embora os profissionalizados tenham uma média ligeiramente superior.

No Quadro 3 apresentam-se os valores médios obtidos relativamente a cada uma das 20 variáveis que compõem o ICAC. Conforme podemos verificar, os itens que obtêm valores médios mais elevados são: 3- Não desistente e 12- Não embirrento. $\mathrm{O}$ item 4- Falador destaca-se pelo seu valor mais baixo. Também o desvio padrão obtido nesse item e no item 15, respectivamente 1,02 e 1,05, sobressaem-se, apontando para uma significativa variação das respostas dadas pelos sujeitos.

\begin{tabular}{|c|c|r|}
\hline Variável & Média & D.P. \\
\hline 1 - Simpático & 3,42 &, 66 \\
\hline 2 - Franco & 4,04 &, 65 \\
\hline 3 - Não desistente & 4,51 &, 70 \\
\hline 4 - Falador & 3,01 & 1,02 \\
\hline 5 - Rápido & 3,27 &, 77 \\
\hline 6 - Tolerante & 3,71 &, 71 \\
\hline 7 - Responsável & 4,39 &, 60 \\
\hline 8 - Enfrentador & 4,21 &, 65 \\
\hline 9 - Aceitável & 3,62 &, 64 \\
\hline 10 - Pragmático & 3,88 &, 69 \\
\hline 11 - Persistente & 3,88 &, 78 \\
\hline 12 - Não-embirrento & 4,59 &, 69 \\
\hline 13 - Verdadeiro & 3,96 &, 83 \\
\hline 14 - Competente & 3,88 &, 63 \\
\hline 15 - Fazer/apetecer & 3,53 & 1,05 \\
\hline 16 - Bem-estar & 3,61 &, 79 \\
\hline 17 - Agradável & 3,58 &, 67 \\
\hline 18 - Não-dependente & 4,00 &, 86 \\
\hline 19 - Bem sucedido & 4,22 &, 67 \\
\hline 20 - Enérgico & 3,57 & \\
\hline
\end{tabular}

QUADRO 3 - MÉDIAS E DESVIO-PADRÃO DOS ITENS 


\section{Análise Factorial de Correspondências}

Foi efectuada a análise factorial de correspondências tomando como activas as 20 variáveis do ICAC e como suplementares as variáveis relativas à identidade dos sujeitos (sexo, idade, categoria, tempo de serviço e tempo como profissionalizado).

Os 5 primeiros factores encontrados explicam $51,05 \%$ da variância. No Quadro 4 e seguintes, é possível ver as variáveis que mais contribuem para a definição desses factores, tanto no lado positivo como negativo do eixo, a percentagem da contribuição de cada uma dessas variáveis para a definição do factor (contribuição absoluta) e a percentagem em que o factor explica a própria variável (contribuição relativa).

O primeiro factor corresponde a $14,8 \%$ da variância. O professor cujo perfil é definido do lado positivo deste factor tem uma ideia positiva acerca da forma como estabelece a sua relação com os outros, tende a considerar-se agradável, simpático, aceitável e tolerante e sente-se bem com a sua forma de ser (bem-estar). As variáveis que se situam do lado negativo do factor são as que se relacionam com a ideia de estabilidade e eficiência (não-desistente, nãodependente, rápido, persistente e enfrentador). Podemos considerar que se trata de um factor de aceitação social em que o indivíduo constrói o seu auto-conceito a partir da forma como vê a sua relação com os outros.

\begin{tabular}{|c|c|c|c|c|c|}
\hline \multicolumn{3}{|c|}{+} & \multicolumn{3}{|c|}{ - } \\
\hline variável & $\begin{array}{l}\text { Contrib. } \\
\text { Absoluta }\end{array}$ & $\begin{array}{l}\text { Contrib. } \\
\text { Relativa }\end{array}$ & variável & $\begin{array}{l}\text { Contrib. } \\
\text { Absoluta }\end{array}$ & $\begin{array}{l}\text { Contrib. } \\
\text { Relativa }\end{array}$ \\
\hline 17- Agradável & 15.7 & 50 & 5- Rápido & 11.4 & 19 \\
\hline 1-Simpático & 14.3 & 38 & $\begin{array}{c}\text { 3-Não desis- } \\
\text { tente }\end{array}$ & 10.0 & 30 \\
\hline 16- Bem-estar & 11.8 & 24 & $\begin{array}{l}\text { 18- Não-depen- } \\
\text { dente }\end{array}$ & 7.3 & 12 \\
\hline 9- Aceitável & 8.7 & 29 & 11- Persistente & 4.8 & 12 \\
\hline 6- Tolerante & 6.4 & 14 & 8- Enfrentador & 3.6 & 15 \\
\hline
\end{tabular}

QUADRO $4-1^{\circ}$ FACTOR DO ICAC 


\begin{tabular}{|c|c|c|c|c|c|}
\hline \multicolumn{2}{|c|}{+} & \multicolumn{3}{|c|}{} \\
\hline Variável & $\begin{array}{c}\text { Contrib. } \\
\text { Absoluta }\end{array}$ & $\begin{array}{c}\text { Contrib. } \\
\text { Relativa }\end{array}$ & variável & $\begin{array}{c}\text { Contrib. } \\
\text { Absoluta }\end{array}$ & $\begin{array}{c}\text { Contrib. } \\
\text { Relativa }\end{array}$ \\
\hline 13- Verdadeiro & 32.8 & 47 & $\begin{array}{c}\text { 18- Não-depen- } \\
\text { dente }\end{array}$ & 22.1 & 29 \\
11- Persistente & 12.8 & 24 & 5- Rápido \\
10- Pragmático & 5.9 & 15 & 16.8 & 22 \\
\hline
\end{tabular}

QUADRO 5 - $2^{\circ}$ FACTOR DO ICAC

\begin{tabular}{|c|c|c|c|c|c|}
\hline \multicolumn{3}{|c|}{+} & \multicolumn{3}{|c|}{ - } \\
\hline variável & $\begin{array}{l}\text { Contrib. } \\
\text { Absoluta }\end{array}$ & $\begin{array}{l}\text { Contrib. } \\
\text { Relativa }\end{array}$ & Variável & $\begin{array}{l}\text { Contrib. } \\
\text { Absoluta }\end{array}$ & $\begin{array}{l}\text { Contrib. } \\
\text { Relativa }\end{array}$ \\
\hline 5- Rápido & 22.2 & 25 & $\begin{array}{l}\text { 18- Não-depen- } \\
\text { dente }\end{array}$ & 25.0 & 28 \\
\hline 6- Tolerante & 8.1 & 12 & 16- Bem-estar & 14.0 & 19 \\
\hline 2- Franco & 7.3 & 15 & 11-Persistente & 7.1 & 11 \\
\hline 13- Verdadeiro & 3.0 & 4 & & & \\
\hline $\begin{array}{c}\text { 19- Bem suce- } \\
\text { dido }\end{array}$ & 2.9 & 6 & & & \\
\hline
\end{tabular}

QUADRO 6 - $3^{\circ}$ FACTOR DO ICAC

O segundo factor (correspondendo a $11,45 \%$ da variância), tem mais a ver com as qualidades intrínsecas que o professor identifica (verdadeiro, persistente e pragmático) e já não tanto as suas qualidades relacionais. No pólo negativo aparecem as duas variáveis (não dependente e rápido) que já estavam do lado negativo no factor 1, mas apresentando agora contribuições para o factor muito mais elevadas. Podemos considerar que se trata de um factor de auto-eficácia ou de resistência interna. Trata-se do grupo de indivíduos que acima de tudo se vêem como verdadeiros, não se preocupando com a sua dependência em relação aos outros ou com a sua rapidez mas, fieis a si próprios, se consideram persistentes e pragmáticos, em oposição a um grupo de indivíduos que se consideram não dependentes e rápidos. 
O terceiro factor explica 9,8\% da variância. As variáveis 5 (rápido) e 18 (não-dependente), que até aqui surgiam juntas para se oporem tanto à aceitaçãosocial como à auto-eficácia, subdividem-se agora em dois padrões: por um lado, temos os indivíduos que valorizam, acima de tudo, o fazerem as coisas de forma mais rápida e imediata, sendo francos, verdadeiros e tolerantes, sem verem nos outros obstáculos à sua eficácia e ao seu sucesso; por outro, os que valorizam a sua independência, o seu bem-estar e afirmação persistente das suas posições. Retomando, mais uma vez, as designações do autor do questionário, podemos designar os primeiros como os impulsivos.

O quarto factor explica 8,36\% da variância, sendo explicado pelas mesmas variáveis do anterior. As variáveis não-dependente e rápido continuam a opor-se. Agora os indivíduos que se consideram independentes consideram-se também tolerantes e verdadeiros (auto-afirmação). Por outro lado, os indivíduos que se vêem como rápidos, sentem-se bem na sua forma de ser e consideram-se persistentes.

O quinto factor explica $6,65 \%$ da variância e o mais importante agora é o sentir-se bem consigo próprio, considerando-se verdadeiro e rápido, por oposição ao perfil dos indivíduos cuja imagem de si mesmos assenta na energia, resistência, simpatia e pragmatismo.

\section{Classificação hierárquica}

Para complementar a informação obtida através da análise de correspondências, foi feita a classificação hierárquica das 20 variáveis do ICAC. Foram utilizados vários métodos de agregação: AVL. (algoritmo de validade da ligação); AMP, SL (critério de ligação única); CL (complete linkage); AVB e AVMR. Os modelos probabilísticos AVL e AVB, baseados no coeficiente de afinidade, mostraram ser os que fornecem mais informação para a análise dos dados.

Utilizando o modelo de agregação AVL (Figura 1), verifica-se que os níveis em que houve maior aumento de informação foram o 18 (nível em que a estatística global atinge o seu máximo absoluto e o acréscimo mais significativo), o 14 e o 10 . 


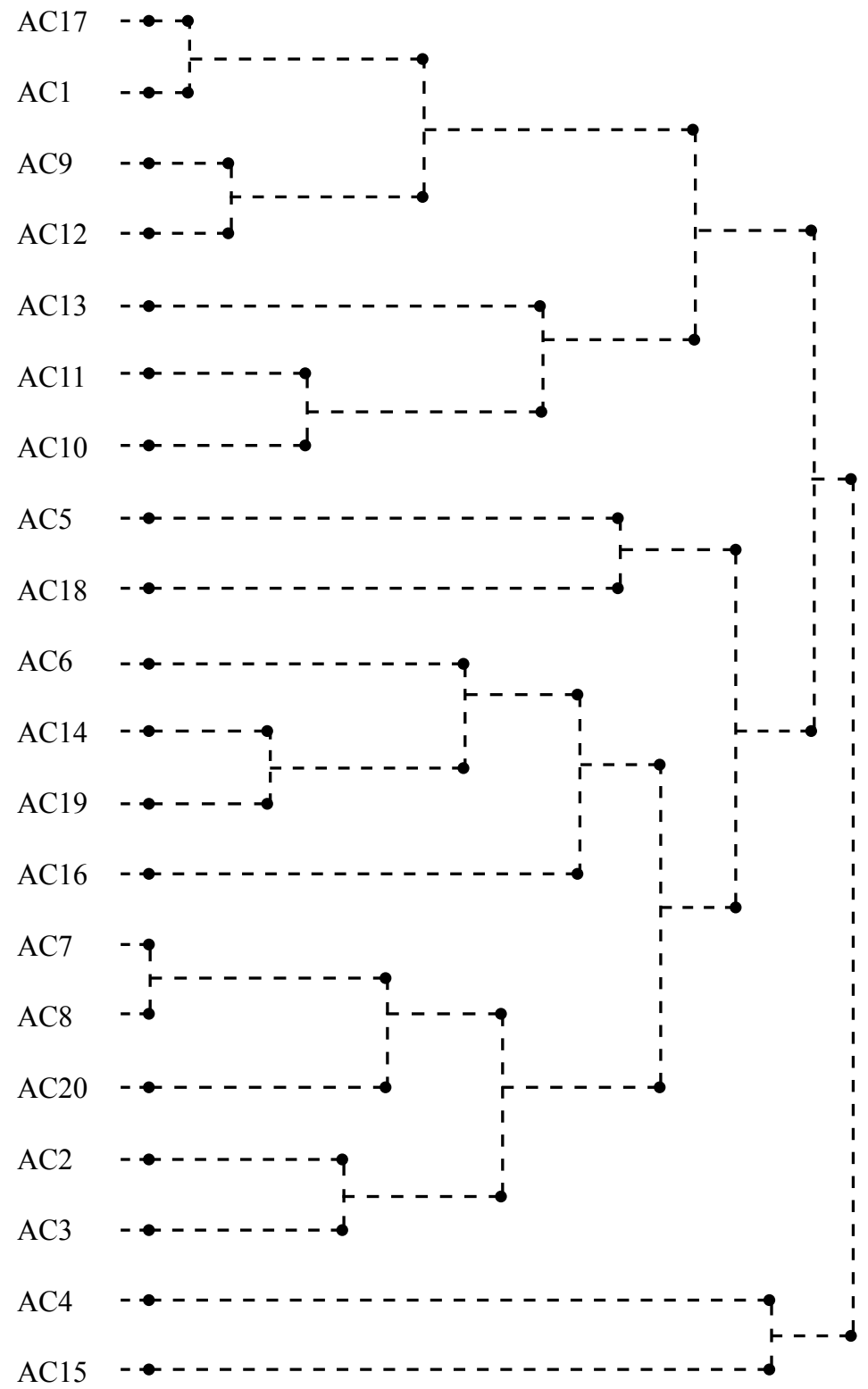

FIGURA 1 - CLASSIFICAÇÃO HIERÁRQUICA DO ICAC - AGREGAÇÃO PELA FAMÍLIA AVL - DENDOGRAMA 
Começando por considerar o nível 10, as variáveis encontram-se agrupadas em 4 grandes grupos: Um primeiro grupo, que caracteriza os professores que vêem a si próprios como sendo agradáveis, simpáticos, aceitáveis e não-embirrentos (correspondentes às variáveis 17, 1, 9 e 12). Trata-se das mesmas variáveis do primeiro factor encontrado na Análise Factorial de Correspondências, tendo a mais a 12 (não-embirrento) e faltando a 16 (bem-estar) e a 6 (tolerante). Um segundo grupo é constituído pelas variáveis 11 e 10 , correspondendo às qualidades persistente e pragmático, ambas também agrupadas no $2^{\circ}$ factor da AFC. A esse segundo grupo juntar-se-á, ao nível 11, a variável 13 (verdadeiro). Um terceiro grupo caracteriza o perfil dos indivíduos que têm uma imagem de si mesmos como sendo competentes, bem-sucedidos e tolerantes (variáveis 14, 19 e 6). Uma quarta classe é constituída a partir das variáveis 7 (responsável), 8 (enfrentador), 20 (enérgico), 2 (franco) e 3 (não-desistente), mais semelhante ao factor impulsividade.

Fazendo o corte ao nível 14, é possível verificar que o primeiro grupo se mantém; ao segundo junta-se a variável 13 (verdadeiro); ao terceiro junta-se a 16 (bem-estar); e surge um novo grupo constituído pelos itens 5 (rápido) e 18 (não-dependente); o quarto não sofre alteração, juntando-se com o terceiro a essse nível. Os itens 4 (falador) e 15 (fazer/apetecer), passados a suplementares na $\mathrm{AFC}$, só se agrupam entre si ao nível 17 e com os restantes ao 19, confirmando a sua fraca ligação com as restantes variáveis.

Usando o método de agregação AVB (Figura 3), os níveis em que houve maior aumento de informação foram 16, 17, 14 e 9. 


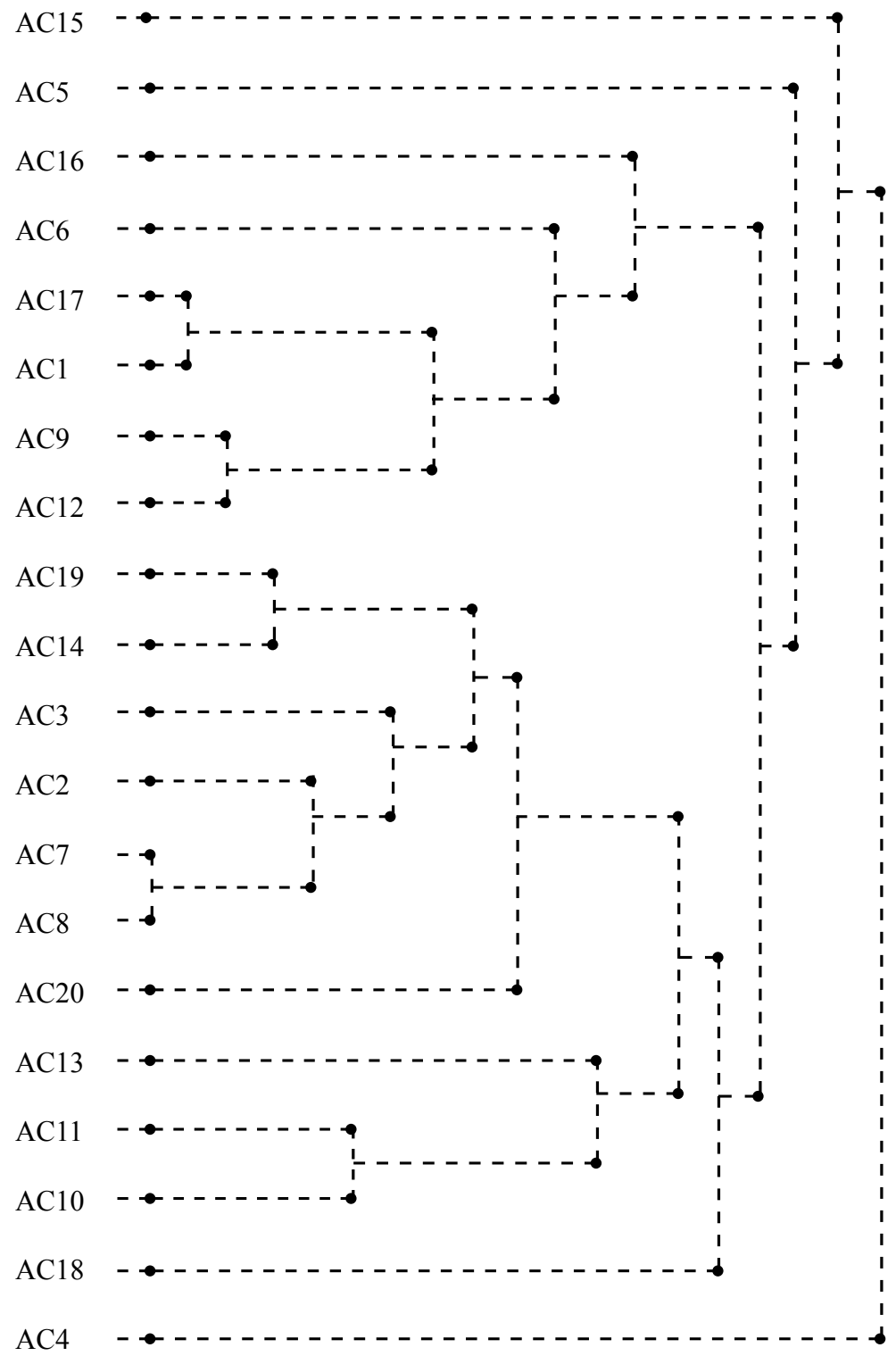

FIGURA 2 - CLASSIFICAÇÃO HIERÁRQUICA DO ICAC - AGREGAÇÃO AVB - DENDOGRAMA 
Fazendo um primeiro corte ao nível 9, verificam-se os seguintes agrupamentos: O primeiro caracteriza, mais uma vez, os indivíduos que têm uma ideia de si mesmos de acordo com as variáveis 17 (agradável), 1 (simpático), 9 (aceitável) e 12 (não embirrento). Esse grupo é exactamente igual ao obtido usando o método AVL. Um segundo grupo identifica os professores que se consideram bem-sucedidos, competentes, enfrentadores, responsáveis, francos e não-desistentes (variáveis 19, 14, 8, 7, 2 e 3) Um terceiro grupo é igual ao já encontrado anteriormente com a agregação AVL, sendo constituído pelas variáveis 11 e 10, correspondendo às qualidades persistente e pragmático.

Ao nível 14, as variáveis 6 (tolerante) e 16 (bem-estar) juntam-se ao primeiro grupo, enquanto que ao segundo se junta a 20 (enérgico) e ao terceiro se volta a juntar a 13 (verdadeiro). Ao nível 16 os grupos 2 e 3 unem-se (mais o item 18-não-dependente) e de seguida unem-se ao primeiro grupo. Ficam de fora: 5,15 e 4 , que apenas a um nível superior se irão juntar.

Verifica-se assim grande concordância entre os resultados obtidos com os dois métodos de agregação.

Das diferentes análises realizadas até aqui, é possível constatar que algumas das variáveis aparecem fortemente associadas independentemente dos métodos utilizados.

Um grupo que podemos designar de aceitação social aglutina: 17- Agradável, 1- Simpático, 9- Aceitável, 12- Não-embirrento, 6- Tolerante, 16- Bemestar.

Um segundo grupo (eficácia ou auto-afirmação): 7- Responsável, 8- Enfrentador, 20- Enérgico.

O terceiro grupo corresponde ao factor auto-eficácia ou resistência interna e inclui as variáveis: 11- Persistente e 10- Pragmático.

As variáveis 5- Rápido, 4- Falador e 15-Fazer/apetecer não aparecem agrupadas.

\section{Discussão e conclusões}

Em termos mais globais, constata-se que existem diferentes perfis de autoconceito nos professores, tal como acontece na população em geral. Os dois mais claramente diferenciados referem-se à aceitação social e auto-eficácia.

Há um grupo de sujeitos cujo autoconceito se alicerça numa visão favorável de si próprios, construída com base naquilo que entendem ser a forma como os outros os vêem em termos relacionais. Consideram-se preponderantemente 
simpáticos, agradáveis e bem aceites pelos outros. Esse é o perfil mais comum entre os professores de categoria profissional mais baixa, não profissionalizados e mais novos. Os indivíduos com esse perfil tendem também a ser mais extrovertidos e a privilegiar as suas qualidades de relação interpessoal no processo de construção do autoconceito. Pelo contrário, os indivíduos com mais tempo de serviço e de profissionalização tendem a apresentar valores mais baixos no autoconceito total e um perfil menos marcado pela aceitação social.

Um segundo perfil de autoconceito liga-se com a auto-eficácia, ou seja, a forma como o indivíduo avalia já não a sua relação mas a sua competência definida pela capacidade para enfrentar e resistir à contrariedades e ser persistente e coerente. A auto-eficácia parece ser relativamente independente das características etárias do professor ou da sua posição na carreira, se bem que professores mais novos, com menos tempo de serviço e categoria profissional mais baixa pareçam estar menos relacionados com esse factor.

A concepção de Markus e Wurf (1987) de autoconceito do momento, que permite compreender o carácter dinâmico do autoconceito, parece ser aqui de extrema utilidade para a compreensão dos dados obtidos. O autoconceito do momento é a configuração particular de representações esboçadas a partir do autoconceito que regulam as acções e reacções actuais do indivíduo.

É a influência do autoconceito do momento que vai marcar e controlar do comportamento tanto nos seus aspectos processos intrapessoais quanto interpessoais (FRANCO, 1999). Os processos intrapessoais mediados pelo autoconceito referem-se nomeadamente ao processamento de informação, à regulação do afecto (envolve habitualmente a defesa do indivíduo contra estados emocionais negativos) e a motivação. Os processos interpessoais, igualmente mediados pelo autoconceito, referem-se à percepção social, à escolha de situações e parceiros, à escolha de estratégias de interacção e às reacções ao feedback.

Assim, o perfil do autoconceito, mais do que o seu valor global, será importante para compreender as diferenças que os professores apresentam na sua prática profissional e na relação que estabelecem com os alunos e com a escola.

\section{REFERÊNCIAS}

BACELAR-NICOLAU, H.; FIGUEIRA, M. L. Análise exploratória da evolução do autoconceito nos adolescentes através de modelos probabilísticos de classificação hierárquica. Psiquiatria Clínica, v. 10, n. 1, p. 43-48, 1989. 
BACELAR-NICOLAU, H.; NICOLAU, F. Estatística e análise de dados multivariada: passado e futuro. Actas do Congresso Anual da Sociedade Portuguesa de Estatística, Lisboa, 1993. p. 521-539.

BACELAR-NICOLAU, H. Contribuições no estudo dos coeficientes de comparação em análise classificatória. Tese de Doutoramento não publicada, Faculdade de Ciências da Universidade de Lisboa. Lisboa, 1980.

BACELAR-NICOLAU, H. The affinity coefficient in cluster analysis Methods of Operations Research. In: MARTIN, J. et al. (Ed.). Munchen: Verlag Anton Hain, 1985. v. 53, p. 507-512.

BACELAR-NICOLAU, H.; DÓRIA, I. O programa $A F I N d e$ classificação hierárquica ascendente - Notas e comunicações. CEAUL (INIC), 1989.

BACELAR-NICOLAU, H.; NICOLAU, F.COSTA. Exploratory and confirmatory discrete multivariate analysis in a probabilistic approach for studying the regional distribution of AIDS in Angola. In: DIDAY, E.; LECHEVALLIER, Y.; SHADER, M. (Eds.). New approaches in classification and data analysis. [S.1.]:Springer-Verlag, 1994. p. 610-618.

CHAN, C. et. al. - Primary School Teachers Self Concept: its relationship with teacher behaviours and student's educational outcomes. Primary Education, v. 3, n. 1, p. 9-28, 1992.

FARIA, M. C. Comunicação individual e comunicação social (a sua relação com o autoconceito, temperamento apoio social e êxito). Dissertação (Mestrado) - Universidade de Coimbra, Faculdade de Psicologia e Ciências da Educação. Coimbra, 1993.

FRANCO, V. Desenvolvimento e personalidade do professor: uma abordagem psicodinâmica do valor transicional dos meios educativos. Évora: Universidade de Évora, 1999.

LEBART, L. ; SALEM, A. Analyse statistique des données textuells. Paris: Dunod, 1988.

LERMAN, I. C. Classification et analyse ordinale des données. Paris: Dunod, 1981.

MARKUS, H.; WURF, E. The dynamic self-concept: a social psychological perspective. Annual Review of Psychology, 38, p.299-337, 1987.

ROCHE, L. A.; MARSH, H. W. Multiple dimensions of university teacher self-concept. Instructional Science, v. 28, n. 5, 2000.

SCHULTZ, E. W.; WOLF, J. Teacher behavior, self-concept and the helping process. 
Psychology in the Schools, v. 10, n. 1, p. 75-78, 2006.

SIMÕES, M. F. O interesse do auto-conceito em educação. Lisboa: Plátano Edições Técnicas, 2001.

TONELSON, S. W. The importance of teacher self concept to create a healthy psychological environment for learning. Education, v. 102, n. 1, p. 96, 1981.

VAZ-SERRA, A. A importância clínica do autoconceito. Psiquiatria Clínica, v. 7, n. 2, p. 17-66. 1986a.

VAZ-SERRA, A. O inventário clínico do autoconceito. Psiquiatria Clínica, v. 7, n. 2, p. 67-84, 1986b.

VAZ-SERRA, A. Atribuição e auto-conceito. Psychologica, v. 1, p. 127-141, 1988.

VAZ-SERRA, A. O interesse clínico das escalas de avaliação de auto-conceito e de coping. Revista de Psicologia Militar, p. 1-17, 1992.

VAZ-SERRA, A.; ANTUNES, R.; FIRMINO, H. Relação entre auto-conceito e expectativas. Psiquiatria Clínica, v. 7, n. 2, p. 85-90, 1986.

VAZ-SERRA, A.; GONÇALVES, S.; FIRMINO, H. Auto-conceito e ansiedade social. Psiquiatria Clínica, v. 7, n. 2, p. 103-108, 1986.

VAZ-SERRA, A.; MATOS, A. P.; GONÇALVES, S. Auto-conceito e sintomas depressivos na população em geral. Psiquiatria Clínica, v. 7, n. 2, p. 97-101, 1986.

VAZ-SERRA, A; RAMALHEIRA, A. C.; FIRMINO, H. Estratégias de coping e autoconceito. Psiquiatria Clínica, v. 9, n. 4, p. 317-322, 1988.

VILLA, A.; CALVETE, E. Development of the teacher self-concept evaluation scale and its relation to burnout. Studies in Educational Evaluation, v. 27, n. 3, p. 239-255, 2001 . 